\title{
Author Index Volume 41 (2014)
}

The issue number is given in front of the pagination

Adriaanse, S., see Zwan, M. (3) 801-807

Aerts, T., see Vermeiren, Y. (3) 819-833

Aguayo, I.S., see Torkamani, M. (2) 515-523

Aguilar, M., see Sánchez-Benavides, G. (3) 887-901

Akatsu, H., see Miyashita (4) 1031-1038

Akazawa, K., see Miyashita (4) 1031-1038

Alawadi, Z., see Al-khateeb, E. (1) 203-211

Al-dalahmah, O., see Al-khateeb, E. (1) 203-211

Alegret, M., G. Cuberas-Borrós, A. Espinosa, S. Valero, I. Hernández, A. Ruíz, J.T. Becker, M. Rosende-Roca, A. Mauleón, O. Sotolongo, J. Castell-Conesa, I. Roca, L. Tárraga and M. Boada, Cognitive, Genetic, and Brain Perfusion Factors Associated with Four Year Incidence of Alzheimer's Disease from Mild Cognitive Impairment (3) 739-748

Al-khateeb, E., E. Al-zayadneh, O. Al-dalahmah, Z. Alawadi, F. khatib, R. Naffa and Y. Shafagoj, Relation between Copper, Lipid Profile, and Cognition in Elderly Jordanians (1) 203-211

Alonso, R., D. Pisa, A.I. Marina, E. Morato, A. Rábano and L. Carrasco, Fungal Infection in Patients with Alzheimer's Disease (1) 301-311

Altamura, C., see Viticchi, G. (2) 401-410

Altavilla, R., see Viticchi, G. (2) 401-410

Al-zayadneh, E., see Al-khateeb, E. (1) 203-211

Amari, M., see Miyashita (4) 1031-1038

Anderson, A., see Brandt, J. (3) 937-945

Anfossi, M., see Borroni, B. (2) 371-376

Annweiler, C., see Mignardot, J.-B. (2) 431-439

Antenora, A., see Testi, S. (3) 709-714

Antúnez, C., see Sánchez-Benavides, G. (3) 887-901

Arai, H., see Miyashita (4) 1031-1038

Arai, H., see Waragai, M. (4) 1207-1222

Arias, C., see Cadena-del-Castillo, C. (3) 845-854

Armstrong, K., see Tarumi, T. (3) 765-778

Arnold, S.E., see Korecka, M. (2) 441-451

Asada, T., see Miyashita (4) 1031-1038

Ashford, J.W. and A.J. Furst, Advancing Brain Imaging for Alzheimer's Disease: Integrating Anatomic and Physiologic Measures (3) 759-763
Bagchi, D., see Khemka, V.K. (2) 525-533

Bagnoli, S., see Evangelisti, E. (1) 289-300

Bai, Z., see Feng, X. (2) 587-597

Bai, Z., see Sun, J. (4) 1039-1056

Ballard, K.J., see Leyton, C.E. (2) 575-585

Bandyopadhyay, K., see Khemka, V.K. (2) 525-533

Bangen, K.J., see Zlatar, Z.Z. (3) 809-817

Barkhof, F., see van der Flier, W.M. (1) 313-327

Bartolini, M., see Viticchi, G. (2) 401-410

Basu, D., see Khemka, V.K. (2) 525-533

Batchelor, J., see Mowszowski, L. (4) 1095-1108

Bathsavanis, A., see Xie, J. (4) 1109-1116

Bayer, A., see Green, C.J. (3) 867-875

Bayer, A., see Tales, A. (3) 655-661

Bayer, A., see Tales, A. (3) 665-666

Beattie, E., see Cohen-Mansfield, J. (1) 15-28

Beauchet, O., see Mignardot, J.-B. (2) 431-439

Becatti, M., see Evangelisti, E. (1) 289-300

Becker, J.T., see Alegret, M. (3) 739-748

Belbin, O., see Campanari, M.-L. (3) 911-924

Belfort, T., see Dourado, M.C.N. (3) 947-956

Benítez-Cardoza, C.G., see Hernández-Rodríguez, M. (4) $1073-1085$

Benito-León, J., see Romero, J.P. (1) 213-221

Ben-Shlomo, Y., see Green, C.J. (3) 867-875

Berly, L., see Blanc, F. (4) 1087-1093

Bermejo-Pareja, F., see Romero, J.P. (1) 213-221

Bermúdez-Rattoni, F., see Cadena-del-Castillo, C. (3) 845-854

Bianchi, M., see Borroni, B. (2) 371-376

Bir, A., see Khemka, V.K. (2) 525-533

Biswas, A., see Khemka, V.K. (2) 525-533

Blanc, F., N. Philippi, B. Cretin, C. Kleitz, L. Berly, B. Jung, S. Kremer, I.J. Namer, F. Sellal, B. Jaulhac and J. de Seze, Lyme Neuroborreliosis and Dementia (4) 1087-1093

Blehar, J., see Brandt, J. (3) 937-945

Blennow, K., see Sun, Q. (3) 877-886

Blitzer, R.D., see Wang, J. (2) 643-650

Boada, M., see Alegret, M. (3) 739-748

Boada, M., see Tajes, M. (1) 273-288 
Boespflug, E.L., J. Eliassen, J. Welge and R. Krikorian, Associative Learning and Regional White Matter Deficits in Mild Cognitive Impairment (2) 421-430

Bokde, A.L.W., see Teipel, S.J. (1) 69-83

Bolognin, S., B. Cozzi, P. Zambenedetti and P. Zatta, Metallothioneins and the Central Nervous System: From a Deregulation in Neurodegenerative Diseases to the Development of New Therapeutic Approaches (1) 29-42

Bonfanti dos Santos, D., see de Oliveira, J. (1) 43-60

Borroni, B., M. Grassi, M. Bianchi, A.C. Bruni, R.G. Maletta, M. Anfossi, D. Pepe, A. Cagnin, P. Caffarra, S. Cappa, F. Clerici, A. Daniele, G.B. Frisoni, D. Galimberti, L. Parnetti, R. Perri, I. Rainero, L. Tremolizzo, M. Turla, O. Zanetti and A. Padovani, Estimating the Inheritance of Frontotemporal Lobar Degeneration in the Italian Population (2) 371-376

Bosch-Morató, M., see Tajes, M. (1) 273-288

Bouwman, F., see Zwan, M. (3) 801-807

Bouwman, F.H., see Olde Rikkert, M.G.M. (1) 261-271

Bouwman, F.H., see van der Flier, W.M. (1) 313-327

Bramanti, P., see Vecchio, F. (1) 113-127

Bramanti, P., see Vecchio, F. (4) 1239-1249

Brandner, S., C. Thaler, N. Lelental, M. Buchfelder, A. Kleindienst, J.M. Maler, J. Kornhuber and P. Lewczuk, Ventricular and Lumbar Cerebrospinal Fluid Concentrations of Alzheimer's Disease Biomarkers in Patients with Normal Pressure Hydrocephalus and Posttraumatic Hydrocephalus (4) 1057-1062

Brandt, J., J. Blehar, A. Anderson and A.L. Gross, Further Validation of the Internet-Based Dementia Risk Assessment (3) 937-945

Bruni, A.C., see Borroni, B. (2) 371-376

Buchfelder, M., see Brandner, S. (4) 1057-1062

Buckwalter, K., see Cohen-Mansfield, J. (1) 15-28

Cadena-del-Castillo, C., C. Valdes-Quezada, F. Carmona-Aldana, C. Arias, F. Bermúdez-Rattoni and F. Recillas-Targa, Age-Dependent Increment of Hydroxymethylation in the Brain Cortex in the Triple-Transgenic Mouse Model of Alzheimer's Disease (3) 845-854

Caffarra, P., see Borroni, B. (2) 371-376

Cagnin, A., see Borroni, B. (2) 371-376

Cai, H.-Y., see Qiao, F. (4) 1165-1176

Campanari, M.-L., M.-S. García-Ayllón, O. Belbin, J. Galcerán, A. Lleó and J. Sáez-Valero, Acetylcholinesterase Modulates Presenilin-1 Levels and $\gamma$-Secretase Activity (3) 911-924
Cappa, S., see Borroni, B. (2) 371-376

Carmona-Aldana, F., see Cadena-del-Castillo, C. (3) 845-854

Carrasco, L., see Alonso, R. (1) 301-311

Carter, A.Y., see Fitz, N.F. (2) 535-549

Carter, R., see Harris, C.J. (1) 179-192

Casals-Coll, M., see Sánchez-Benavides, G. (3) 887901

Cascella, R., see Evangelisti, E. (1) 289-300

Caselli, A., see Evangelisti, E. (1) 289-300

Castagna, A., see Gareri, P. (2) 633-640

Castell-Conesa, J., see Alegret, M. (3) 739-748

Castellví, M., see Rami, L. (2) 453-466

Castranio, E.L., see Fitz, N.F. (2) 535-549

Cecchi, C., see Evangelisti, E. (1) 289-300

Cedazo-Mínguez, Á., see Lodeiro, M. (1) 223-232

Cha, M.-Y., see Moon, M. (1) 233-241

Chakrabarti, S., see Khemka, V.K. (2) 525-533

Chan, Q., see Ka-Fung Mak, H. (3) 749-758

Chang, Q., see Yu, Y.-Z. (1) 243-260

Chattopadhyay, M., see Khemka, V.K. (2) 525-533

Chen, A., see Yu, Y.-Z. (1) 243-260

Chen, C., see Song, G. (1) 85-99

Chen, W.-T., see Shih, Y.-H. (3) 855-865

Chen, Y., see Zhou, X. (3) 925-935

Choi, J.G., see Moon, M. (2) 599-613

Choi, K.-G., see Kim, G.H. (1) 169-178

Chu, J., E. Lauretti, C.P. Craige and D. Praticò, Pharmacological Modulation of GSAP Reduces Amyloid- $\beta$ Levels and Tau Phosphorylation in a Mouse Model of Alzheimer's Disease with Plaques and Tangles (3) 729-737

Chu, J., see Lin, L. (4) 1149-1163

Chu, L.W., see Ka-Fung Mak, H. (3) 749-758

Cifuentes, A., see Lodeiro, M. (1) 223-232

Clarimón, J., see Tajes, M. (1) 273-288

Clerici, F., see Borroni, B. (2) 371-376

Cockayne, N., see Mowszowski, L. (4) 1095-1108

Cohen-Mansfield, J., K. Buckwalter, E. Beattie, K. Rose, C. Neville and A. Kolanowski, Expanded Review Criteria: The Case of Nonpharmacological Interventions in Dementia (1) 15-28

Cornu, C., see Mignardot, J.-B. (2) 431-439

Correa-Basurto, J., see Hernández-Rodríguez, M. (4) 1073-1085

Coskuner, O. and I.V.J. Murray, Adenosine Triphosphate (ATP) Reduces Amyloid- $\beta$ Protein Misfolding in vitro (2) 561-574

Cotroneo, A.M., see Gareri, P. (2) 633-640

Cozzi, B., see Bolognin, S. (1) 29-42

Crack, P.J., see Feng, X. (2) 587-597 
Craige, C.P., see Chu, J. (3) 729-737

Cretin, B., see Blanc, F. (4) 1087-1093

Cuadrado, M.L., see Garcia-Ptacek, S. (2) 467-477

Cuberas-Borrós, G., see Alegret, M. (3) 739-748

Cullum, C.M., see Tarumi, T. (3) 765-778

Dale, A.M., see Engvig, A. (3) 779-791

Dam, D.V., see Vermeiren, Y. (3) 819-833

Daniele, A., see Borroni, B. (2) 371-376

Darsalia, V., see Hussain, S. (2) 551-560

Dauphinot, V., see Xie, J. (4) 1109-1116

Dautzenberg, P.L.J., see Olde Rikkert, M.G.M. 261-271

De Deyn, P.P., see Struyfs, H. (3) 903-909

De Deyn, P.P., see Vermeiren, Y. (3) 819-833

de Oliveira, J., E.L.G. Moreira, D. Bonfanti dos Santos, T.C. Piermartiri, R.C. Dutra, S. Pinton, C.I. Tasca, M. Farina, R.D.S. Prediger and A. Fabro de Bem, Increased Susceptibility to Amyloid- $\beta$-Induced Neurotoxicity in Mice Lacking the Low-Density Lipoprotein Receptor (1) 43-60

de Seze, J., see Blanc, F. (4) 1087-1093

Delphin-Combe, F., see Xie, J. (4) 1109-1116

Deschamps, T., see Mignardot, J.-B. (2) 431-439

Desestret, V., see Xie, J. (4) 1109-1116

Diamond, K., see Mowszowski, L. (4) 1095-1108

DidierLaurent, G., see Sauvée, M. (2) 377-386

Dlugaj, M., G. Weinreich, C. Weimar, A. Stang, N. Dragano, T.E. Wessendorf, H. Teschler, A. Winkler, N. Wege, S. Moebus, S. Möhlenkamp, R. Erbel, K.-H. Jöckel and on behalf of the Heinz Nixdorf Recall Study Investigative Group, SleepDisordered Breathing, Sleep Quality, and Mild Cognitive Impairment in the General Population (2) 479-497

Dong, Y., see Shao, H. (2) 499-513

Doody, R.S., see Szigeti, K. (4) 1063-1071

Dorey, A., see Xie, J. (4) 1109-1116

Dourado, M.C.N., D.C. Mograbi, R.L. Santos, M.F.B. Sousa, M.L. Nogueira, T. Belfort, J. LandeiraFernandez and J. Laks, Awareness of Disease in Dementia: Factor Structure of the Assessment Scale of Psychosocial Impact of the Diagnosis of Dementia (3) 947-956

Dragano, N., see Dlugaj, M. (2) 479-497

Duan, Y., see Feng, X. (2) 587-597

Duan, Y., see Sun, J. (4) 1039-1056

Due-Tønnessen, P., see Engvig, A. (3) 779-791

Dunsky, D.I., see Tarumi, T. (3) 765-778

Dutra, R.C., see de Oliveira, J. (1) 43-60

Dyrba, M., see Teipel, S.J. (1) 69-83
Ebrahim, S., see Green, C.J. (3) 867-875

Eliassen, J., see Boespflug, E.L. (2) 421-430

Engelborghs, S., see Struyfs, H. (3) 903-909

Engelborghs, S., see Vermeiren, Y. (3) 819-833

Engvig, A., A.M. Fjell, L.T. Westlye, N.V. Skaane, A.M. Dale, D. Holland, P. Due-Tønnessen, Ø. Sundseth and K.B. Walhovd, Effects of Cognitive Training on Gray Matter Volumes in Memory Clinic Patients with Subjective Memory Impairment (3) 779-791

Eraso-Pichot, A., see Tajes, M. (1) 273-288

Erbel, R., see Dlugaj, M. (2) 479-497

Eriksdotter, M., see Eriksson, H. (2) 411-419

Eriksdotter, M., see Garcia-Ptacek, S. (2) 467-477

Eriksson, H., S.-M. Fereshtehnejad, F. Falahati, B. Farahmand, D. Religa and M. Eriksdotter, Differences in Routine Clinical Practice between Early and Late Onset Alzheimer's Disease: Data from the Swedish Dementia Registry (SveDem) (2) 411-419

Escanyé, M.-C., see Sauvée, M. (2) 377-386

Espinosa, A., see Alegret, M. (3) 739-748

Ethell, D.W., Disruption of Cerebrospinal Fluid Flow through the Olfactory System May Contribute to Alzheimer's Disease Pathogenesis (4) 1021-1030

Evangelisti, E., M. Zampagni, R. Cascella, M. Becatti, C. Fiorillo, A. Caselli, S. Bagnoli, B. Nacmias and C. Cecchi, Plasma Membrane Injury Depends on Bilayer Lipid Composition in Alzheimer's Disease (1) 289-300

Fabbo, A., see Gareri, P. (2) 633-640

Fabrizi, G.M., see Testi, S. (3) 709-714

Fabro de Bem, A., see de Oliveira, J. (1) 43-60

Falahati, F., E. Westman and A. Simmons, Multivariate Data Analysis and Machine Learning in Alzheimer's Disease with a Focus on Structural Magnetic Resonance Imaging (3) 685-708

Falahati, F., see Eriksson, H. (2) 411-419

Falsetti, L., see Viticchi, G. (2) 401-410

Fan, X., see Yang, M. (4) 1131-1147

Farahmand, B., see Eriksson, H. (2) 411-419

Farahmand, B., see Garcia-Ptacek, S. (2) 467-477

Farina, M., see de Oliveira, J. (1) 43-60

Fellgiebel, A., see Teipel, S.J. (1) 69-83

Feng, L., see Ng, T.P. (1) 61-68

Feng, X., see Sun, J. (4) 1039-1056

Feng, X., Z. Bai, J. Wang, B. Xie, J. Sun, G. Han, F. Song, P.J. Crack, Y. Duan and H. Lei, Robust Gene Dysregulation in Alzheimer's Disease Brains (2) 587-597 
Fereshtehnejad, S.-M., see Eriksson, H. (2) 411-419 Fernández-Martínez, M., see Sánchez-Benavides, G. (3) 887-901

Ferrarini, M., see Testi, S. (3) 709-714

Figurski, M., see Korecka, M. (2) 441-451

Filippi, M., see Teipel, S.J. (1) 69-83

Filla, A., see Testi, S. (3) 709-714

Fiorillo, C., see Evangelisti, E. (1) 289-300

Fish, M., see Green, C.J. (3) 867-875

Fitz, N.F., E.L. Castranio, A.Y. Carter, R. Kodali, I. Lefterov and R. Koldamova, Improvement of Memory Deficits and Amyloid- $\beta$ Clearance in Aged APP23 Mice Treated with a Combination of Anti-Amyloid- $\beta$ Antibody and LXR Agonist (2) 535-549

Fjell, A.M., see Engvig, A. (3) 779-791

Forgione, L., see Gareri, P. (2) 633-640

Frahler, K., see Harris, C.J. (1) 179-192

Frank-García, A., see Sánchez-Benavides, G. (3) 887901

Frisardi, V., Impact of Metabolic Syndrome on Cognitive Decline in Older Age: Protective or Harmful, Where is the Pitfall? (1) 163-167

Frisoni, G.B., see Borroni, B. (2) 371-376

Frisoni, G.B., see Teipel, S.J. (1) 69-83

Froestl, W., A. Muhs and A. Pfeifer, Cognitive Enhancers (Nootropics). Part 1: Drugs interacting with Receptors. Update 2014 (4) 961-1019

Fu, Y., Z. Rusznák, J.B.J. Kwok, W.S. Kim and G. Paxinos, Age-Dependent Alterations of the Hippocampal Cell Composition and Proliferative Potential in the hAßPPSwInd-J20 Mouse (4) 1177-1192

Fujii, C., see Waragai, M. (4) 1207-1222

Furst, A.J., see Ashford, J.W. (3) 759-763

Furukawa, K., see Miyashita (4) 1031-1038

Gabelle, A., see Xie, J. (4) 1109-1116

Galcerán, J., see Campanari, M.-L. (3) 911-924

Galimberti, D., see Borroni, B. (2) 371-376

Gallacher, J., see Green, C.J. (3) 867-875

Gao, X.-P., see Qiao, F. (4) 1165-1176

García-Ayllón, M.-S., see Campanari, M.-L. (3) 911-924

Garcia-Ptacek, S., B. Farahmand, I. Kåreholt, D. Religa, M.L. Cuadrado and M. Eriksdotter, Mortality Risk after Dementia Diagnosis by Dementia Type and Underlying Factors: A Cohort of 15,209 Patients based on the Swedish Dementia Registry (2) 467 477

García-Sanchez, C., see Rami, L. (2) 453-466

Gard, P., see Nelson, L. (2) 331-343
Gareri, P., D. Putignano, A. Castagna, A.M. Cotroneo, G.D. Palo, A. Fabbo, L. Forgione, A. Giacummo, R. Lacava, S. Marino, M. Simone, A. Zurlo and S. Putignano, Retrospective Study on the Benefits of Combined Memantine and cholinEsterase inhibitor treatMent in AGEd Patients affected with Alzheimer's Disease: The MEMAGE Study (2) 633-640

Garnier-Crussard, A., see Xie, J. (4) 1109-1116

Gentleman, S.M., see Zhao, Y. (3) 835-844

Giacummo, A., see Gareri, P. (2) 633-640

Giannakopoulos, P., see Haller, S. (1) 101-112

Gil-Gómez, G., see Tajes, M. (1) 273-288

Gómez-Ansón, B., see Sánchez-Benavides, G. (3) 887901

Gong, B., see Wang, J. (2) 643-650

Gramunt, N., see Sánchez-Benavides, G. (3) 887-901

Grassi, M., see Borroni, B. (2) 371-376

Green, C.J., J.M.P. Holly, A. Bayer, M. Fish, S. Ebrahim, J. Gallacher and Y. Ben-Shlomo, The Role of IGF-I, IGF-II, and IGFBP-3 in Male Cognitive Aging and Dementia Risk: The Caerphilly Prospective Study (3) 867-875

Gross, A.L., see Brandt, J. (3) 937-945

Grossman, M., see Korecka, M. (2) 441-451

Grothe, M.J., see Teipel, S.J. (1) 69-83

Guivernau, B., see Tajes, M. (1) 273-288

Guix, F.X., see Tajes, M. (1) 273-288

Guo, X., see Zhao, Y. (3) 835-844

Haller, S., M.-L. Montandon, C. Rodriguez, D. Moser, S. Toma, J. Hofmeister, I. Sinanaj, K.-O. Lovblad and P. Giannakopoulos, Acute Caffeine Administration Effect on Brain Activation Patterns in Mild Cognitive Impairment (1) 101-112

Hamaguchi, T., see Miyashita (4) 1031-1038

Hampel, H., see Sun, Q. (3) 877-886

Hampel, H., see Teipel, S.J. (1) 69-83

Han, G., see Feng, X. (2) 587-597

Han, G., see Sun, J. (4) 1039-1056

Hanyu, H., see Miyashita (4) 1031-1038

Harigaya, Y., see Miyashita (4) 1031-1038

Haritou, M., see Torkamani, M. (2) 515-523

Harris, C.J., K. Voss, C. Murchison, M. Ralle, K. Frahler, R. Carter, A. Rhoads, B. Lind, E. Robinson and J.F. Quinn, Oral Zinc Reduces Amyloid Burden in Tg2576 Mice (1) 179-192

Harrison, S.L., see Siervo, M. (1) 151-161

Hartikainen, S., see Taipale, H. (4) 1223-1228

Hartmann, T., N. van Wijk, R.J. Wurtman, M.G.M. Olde Rikkert, J.W.C. Sijben, H. Soininen, B. 
Vellas and P. Scheltens, A Nutritional Approach to Ameliorate Altered Phospholipid Metabolism in Alzheimer's Disease (3) 715-717

Hata, S., see Waragai, M. (4) 1207-1222

Hatsuta, H., see Miyashita (4) 1031-1038

Hauenstein, K., see Teipel, S.J. (1) 69-83

He, R., see Yang, M. (4) 1131-1147

Hea, R., see Yang, M. (4) 1117-1129

Hermens, D.F., see Mowszowski, L. (4) 1095-1108

Hernández, I., see Alegret, M. (3) 739-748

Hernández-Rodríguez, M., J. Correa-Basurto, F. Martínez-Ramos, I.I. Padilla-Martínez, C.G. Benítez-Cardoza, E. Mera-Jiménez and M.C. Rosales-Hernández, Design of Multi-Target Compounds as AChE, BACE1, and Amyloid- $\beta_{1-42}$ Oligomerization Inhibitors: In Silico and In Vitro Studies (4) 1073-1085

Hickie, I.B., see Mowszowski, L. (4) 1095-1108

Higuchi, S., see Miyashita (4) 1031-1038

Higuchi, S., see Waragai, M. (4) 1207-1222

Hill, C., see Tarumi, T. (3) 765-778

Hodges, J.R., see Leyton, C.E. (2) 575-585

Hofmeister, J., see Haller, S. (1) 101-112

Holland, D., see Engvig, A. (3) 779-791

Holly, J.M.P., see Green, C.J. (3) 867-875

Hook, G., J. Yu, T. Toneff, M. Kindy and V. Hook, Brain Pyroglutamate Amyloid- $\beta$ is Produced by Cathepsin B and is Reduced by the Cysteine Protease Inhibitor E64d, Representing a Potential Alzheimer's Disease Therapeutic (1) 129-149

Hook, V., see Hook, G. (1) 129-149

$\mathrm{Hu}$, X., see Yang, M. (4) 1117-1129

$\mathrm{Hu}$, X., see Yang, M. (4) 1131-1147

Huang, L., see Zhang, L. (4) 1193-1205

Huma, T., see Yang, M. (4) 1131-1147

Hurst, W.J., see Wang, J. (2) 643-650

Hussain, S., S. Mansouri, Å. Sjöholm, C. Patrone and V. Darsalia, Evidence for Cortical Neuronal Loss in Male Type 2 Diabetic Goto-Kakizaki Rats (2) 551-560

Hyman, B.T., see Marshall, G.A. (3) 719-728

Ibáñez, C., see Lodeiro, M. (1) 223-232

Igarashi, K., see Waragai, M. (4) 1207-1222

Ihara, Y., see Miyashita (4) 1031-1038

Ikeda, M., see Miyashita (4) 1031-1038

Ikeuchi, T., see Miyashita (4) 1031-1038

Imagawa, M., see Miyashita (4) 1031-1038

Irish, M., see Leyton, C.E. (2) 575-585

Ishii, R., see Waragai, M. (4) 1207-1222

Iwai, N., see Waragai, M. (4) 1207-1222
Jagger, C., see Siervo, M. (1) 151-161

Jahanshahi, M., see Torkamani, M. (2) 515-523

Jak, A.J., see Zlatar, Z.Z. (3) 809-817

Jaulhac, B., see Blanc, F. (4) 1087-1093

Jeong, J.H., see Kim, G.H. (1) 169-178

Jia, J., see Sun, J. (4) 1039-1056

Jiang, Q., see Lin, L. (4) 1149-1163

Jiang, T., see Tan, C.-C. (2) 615-631

Jimenez, E., see Lee, G.J. (3) 793-800

Jöckel, K.-H., see Dlugaj, M. (2) 479-497

Johnson, K.A., see Marshall, G.A. (3) 719-728

Jula, A., see Scheinin, N.M. (1) 193-202

Jung, B., see Blanc, F. (4) 1087-1093

Ka-Fung Mak, H., W. Qian, K.S. Ng, Q. Chan, Y.-Q. Song, L.W. Chu and K. Kai-Wing Yau, Combination of MRI Hippocampal Volumetry and Arterial Spin Labeling MR Perfusion at 3-Tesla Improves the Efficacy in Discriminating Alzheimer's Disease from Cognitively Normal Elderly Adults (3) 749-758

Kai-Wing Yau, K., see Ka-Fung Mak, H. (3) 749-758

Kakita, A., see Miyashita (4) 1031-1038

Kanazawa, I., see Miyashita (4) 1031-1038

Kanios, C., see Torkamani, M. (2) 515-523

Kapila, A.K., H.R. Watts, T. Wang and D. Ma, The Impact of Surgery and Anesthesia on Post-Operative Cognitive Decline and Alzheimer's Disease Development: Biomarkers and Preventive Strategies (1) $1-13$

Kåreholt, I., see Garcia-Ptacek, S. (2) 467-477

Katsanou, M.-N., see Torkamani, M. (2) 515-523

Kawarabayashi, T., see Miyashita (4) 1031-1038

Kawase, Y., see Miyashita (4) 1031-1038

Kellermayer, B., see Szigeti, K. (4) 1063-1071

Khan, M.A., see Tarumi, T. (3) 765-778

khatib, F., see Al-khateeb, E. (1) 203-211

Khemka, V.K., D. Bagchi, K. Bandyopadhyay, A. Bir, M. Chattopadhyay, A. Biswas, D. Basu and S. Chakrabarti, Altered Serum Levels of Adipokines and Insulin in Probable Alzheimer's Disease (2) 525-533

Kim, G.H., J.-E. Kim, K.-G. Choi, S.M. Lim, J.-M. Lee, D.L. Na and J.H. Jeong, T1-weighted Axial Visual Rating Scale for an Assessment of Medial Temporal Atrophy in Alzheimer's Disease (1) 169-178

Kim, J.-E., see Kim, G.H. (1) 169-178

Kim, S.Y., see Moon, M. (2) 599-613

Kim, W.S., see Fu, Y. (4) 1177-1192

Kindy, M., see Hook, G. (1) 129-149

Kitamura, N., see Miyashita (4) 1031-1038 
Kleindienst, A., see Brandner, S. (4) 1057-1062

Kleitz, C., see Blanc, F. (4) 1087-1093

Klöppel, S., see Teipel, S.J. (1) 69-83

Kodali, R., see Fitz, N.F. (2) 535-549

Kolanowski, A., see Cohen-Mansfield, J. (1) 15-28

Koldamova, R., see Fitz, N.F. (2) 535-549

Koncarevic, S., see Russell, C.L. (2) 345-364

Koponen, M., see Taipale, H. (4) 1223-1228

Korecka, M., T. Waligorska, M. Figurski, J.B. Toledo, S.E. Arnold, M. Grossman, J.Q. Trojanowski and L.M. Shaw, Qualification of a Surrogate Matrix-Based Absolute Quantification Method for Amyloid- $\beta_{42}$ in Human Cerebrospinal Fluid Using 2D UPLC-Tandem Mass Spectrometry (2) 441451

Kornhuber, J., see Brandner, S. (4) 1057-1062

Krajcovicova, L., M. Mikl, R. Marecek and I. Rektorova, Disturbed Default Mode Network Connectivity Patterns in Alzheimer's Disease Associated with Visual Processing (4) 1229-1238

Kremer, S., see Blanc, F. (4) 1087-1093

Krikorian, R., see Boespflug, E.L. (2) 421-430

Krolak-Salmon, P., see Xie, J. (4) 1109-1116

Krsteska, R., see Mukaetova-Ladinska, E.B. (4) 12511260

Kuo, Y.-M., see Shih, Y.-H. (3) 855-865

Kuwano, R., see Miyashita (4) 1031-1038

Kwok, J.B.J., see Fu, Y. (4) 1177-1192

Lacava, R., see Gareri, P. (2) 633-640

Laks, J., see Dourado, M.C.N. (3) 947-956

Lal, D., see Szigeti, K. (4) 1063-1071

Lammertsma, A., see Zwan, M. (3) 801-807

Landeira-Fernandez, J., see Dourado, M.C.N. (3) 947956

Lansink, M., see Olde Rikkert, M.G.M. (1) 261-271

Latarche, C., see Sauvée, M. (2) 377-386

Lauretti, E., see Chu, J. (3) 729-737

Lee, C.-W., see Shih, Y.-H. (3) 855-865

Lee, G.J., P.H. Lu, M.J. Mather, J. Shapira, E. Jimenez, A.D. Leow, P.M. Thompson and M.F. Mendez, Neuroanatomical Correlates of Emotional Blunting in Behavioral Variant Frontotemporal Dementia and Early-Onset Alzheimer's Disease (3) 793-800

Lee, J.-M., see Kim, G.H. (1) 169-178

Lee, S., see Taddeo, M.A. (2) 365-369

Lee, T.S., see $\mathrm{Ng}$, T.P. (1) 61-68

Lees, A.J., see Torkamani, M. (2) 515-523

Lefterov, I., see Fitz, N.F. (2) 535-549

Lehmann, S., see Xie, J. (4) 1109-1116
Lei, H., see Feng, X. (2) 587-597

Lei, H., see Sun, J. (4) 1039-1056

Lelental, N., see Brandner, S. (4) 1057-1062

Lemstra, A.W., see van der Flier, W.M. (1) 313-327

Lentini, J.M., see Szigeti, K. (4) 1063-1071

Leow, A.D., see Lee, G.J. (3) 793-800

Levey, A., see Sun, Q. (3) 877-886

Levine, S., see Wang, J. (2) 643-650

Lewczuk, P., see Brandner, S. (4) 1057-1062

Lewis, S.J.G., see Mowszowski, L. (4) 1095-1108

Leyton, C.E., S. Savage, M. Irish, S. Schubert, O. Piguet, K.J. Ballard and J.R. Hodges, Verbal Repetition in Primary Progressive Aphasia and Alzheimer's Disease (2) 575-585

Li, R., see Sun, Q. (3) 877-886

Li, T., see Yang, M. (4) 1131-1147

Lim, S.M., see Kim, G.H. (1) 169-178

Limousin, P.D., see Torkamani, M. (2) 515-523

Lin, L., S.-S. Yang, J. Chu, L. Wang, L.-N. Ning, T. Zhang, Q. Jiang, Q. Tian and J.-Z. Wang, RegionSpecific Expression of Tau, Amyloid- $\beta$ Protein Precursor, and Synaptic Proteins at Physiological Condition or Under Endoplasmic Reticulum Stress in Rats (4) 1149-1163

Lind, B., see Harris, C.J. (1) 179-192

Lista, S., see Sun, Q. (3) 877-886

Liu, C., see Zhang, L. (4) 1193-1205

Liu, J., see Tarumi, T. (3) 765-778

Liu, Q., see Song, G. (1) 85-99

Liu, S., see Szigeti, K. (4) 1063-1071

Liu, S., see Yu, Y.-Z. (1) 243-260

Liu, T.T., see Zlatar, Z.Z. (3) 809-817

Lleó, A., see Campanari, M.-L. (3) 911-924

Lobo, N., see Zhao, Y. (3) 835-844

Locascio, J.J., see Marshall, G.A. (3) 719-728

Lodeiro, M., C. Ibáñez, A. Cifuentes, C. Simó and Á. Cedazo-Mínguez, Decreased Cerebrospinal Fluid Levels of L-Carnitine in Non-Apolipoprotein E4 Carriers at Early Stages of Alzheimer's Disease (1) 223-232

Lorius, N., see Marshall, G.A. (3) 719-728

Louis, E.D., see Romero, J.P. (1) 213-221

Lovblad, K.-O., see Haller, S. (1) 101-112

Lu, J., see Yang, M. (4) 1117-1129

Lü, L., see Yang, M. (4) 1131-1147

Lu, P.H., see Lee, G.J. (3) 793-800

Luzzi, S., see Viticchi, G. (2) 401-410

Ma, C., see Szigeti, K. (4) 1063-1071

Ma, D., see Kapila, A.K. (1) 1-13

Ma, D., see Zhao, Y. (3) 835-844 
Ma, T., see Zhou, X. (3) 925-935

Ma, Y., see Yang, M. (4) 1117-1129

Ma, Y., see Yang, M. (4) 1131-1147

Madeley, L., see Torkamani, M. (2) 515-523

Makazlieva, T., see Mukaetova-Ladinska, E.B. (4) $1251-1260$

Malaplate-Armand, C., see Sauvée, M. (2) 377-386

Maler, J.M., see Brandner, S. (4) 1057-1062

Maletta, R.G., see Borroni, B. (2) 371-376

Mansouri, S., see Hussain, S. (2) 551-560

Marecek, R., see Krajcovicova, L. (4) 1229-1238

Marina, A.I., see Alonso, R. (1) 301-311

Marino, S., see Gareri, P. (2) 633-640

Marra, C., see Vecchio, F. (1) 113-127

Marshall, G.A., N. Lorius, J.J. Locascio, B.T. Hyman, D.M. Rentz, K.A. Johnson and R.A. Sperling for the Alzheimer's Disease Neuroimaging Initiative, Regional Cortical Thinning and Cerebrospinal Biomarkers Predict Worsening Daily Functioning Across the Alzheimer's Disease Spectrum (3) 719-728

Martin, J.-J., see Struyfs, H. (3) 903-909

Martin-Cook, K., see Tarumi, T. (3) 765-778

Martínez-Parra, C., see Sánchez-Benavides, G. (3) 887901

Martínez-Ramos, F., see Hernández-Rodríguez, M. (4) 1073-1085

Mather, M.J., see Lee, G.J. (3) 793-800

Matsubara, E., see Miyashita (4) 1031-1038

Mattace-Raso, F., Is Memantine + Acetylcholinesterase Inhibitor Treatment Superior to Either Therapy Alone in Alzheimer's Disease? (2) 641-642

Mauleón, A., see Alegret, M. (3) 739-748

McDonald, L., see Torkamani, M. (2) 515-523

Meindl, T., see Teipel, S.J. (1) 69-83

Mendez, M.F., see Lee, G.J. (3) 793-800

Meng, X.-F., see Tan, C.-C. (2) 615-631

Mera-Jiménez, E., see Hernández-Rodríguez, M. (4) 1073-1085

Mercier, B., see Xie, J. (4) 1109-1116

Miao, J., see Yang, M. (4) 1117-1129

Miao, J., see Yang, M. (4) 1131-1147

Mignardot, J.-B., O. Beauchet, C. Annweiler, C. Cornu and T. Deschamps, Postural Sway, Falls, and Cognitive Status: A Cross-Sectional Study among Older Adults (2) 431-439

Mikl, M., see Krajcovicova, L. (4) 1229-1238

Miraglia, F., see Vecchio, F. (1) 113-127

Miraglia, F., see Vecchio, F. (4) 1239-1249

Miscione, G.P., see Tajes, M. (1) 273-288
Miyashita, A., Y. Wen, N. Kitamura, E. Matsubara, T. Kawarabayashi, M. Shoji, N. Tomita, K. Furukawa, H. Arai, T. Asada, Y. Harigaya, M. Ikeda, M. Amari, H. Hanyu, S. Higuchi, M. Nishizawa, M. Suga, Y. Kawase, H. Akatsu, M. Imagawa, T. Hamaguchi, M. Yamada, T. Morihara, M. Takeda, T. Takao, K. Nakata, K. Sasaki, K. Watanabe, K. Nakashima, K. Urakami, T. Ooya, M. Takahashi, T. Yuzuriha, K. Serikawa, S. Yoshimoto, R. Nakagawa, Y. Saito, H. Hatsuta, S. Murayama, A. Kakita, H. Takahashi, H. Yamaguchi, K. Akazawa, I. Kanazawa, Y. Ihara, T. Ikeuchi and R. Kuwano, Lack of Genetic Association Between TREM2 and Late-Onset Alzheimer's Disease in a Japanese Population (4) 1031-1038

Moebus, S., see Dlugaj, M. (2) 479-497

Mograbi, D.C., see Dourado, M.C.N. (3) 947-956

Möhlenkamp, S., see Dlugaj, M. (2) 479-497

Molina, H., see Tajes, M. (1) 273-288

Molinuevo, J.L., see Rami, L. (2) 453-466

Molinuevo, J.L., see Sánchez-Benavides, G. (3) 887901

Molinuevo, J.L., see Struyfs, H. (3) 903-909

Mollica, M.A., see Rami, L. (2) 453-466

Montandon, M.-L., see Haller, S. (1) 101-112

Mook-Jung, I., see Moon, M. (1) 233-241

Moon, M., J.G. Choi, S.Y. Kim and M.S. Oh, Bombycis excrementum Reduces Amyloid- $\beta$ OligomerInduced Memory Impairments, Neurodegeneration, and Neuroinflammation in Mice (2) 599-613

Moon, M., M.-Y. Cha and I. Mook-Jung, Impaired Hippocampal Neurogenesis and its Enhancement with Ghrelin in 5XFAD Mice (1) 233-241

Morato, E., see Alonso, R. (1) 301-311

Moreira, E.L.G., see de Oliveira, J. (1) 43-60

Morihara, T., see Miyashita (4) 1031-1038

Moriya, M., see Waragai, M. (4) 1207-1222

Moser, D., see Haller, S. (1) 101-112

Mowszowski, L., D.F. Hermens, K. Diamond, L. Norrie, N. Cockayne, P.B. Ward, I.B. Hickie, S.J.G. Lewis, J. Batchelor and S.L. Naismith, Cognitive Training Enhances Pre-Attentive Neurophysiological Responses in Older Adults 'At Risk' of Dementia (4) 1095-1108

Muhs, A., see Froestl, W. (4) 961-1019

Mukaetova-Ladinska, E.B., R. Krsteska, O. Vaskova, T. Makazlieva and M. Tsolaki, Dementia and Depression in Older Adults: A Southeast European Perspective: Summary of a Dementia 
Psychogeriatric Symposium held in

Macedonia, 23 May 2013 (4) 1251-1260

Muñoz, F.J., see Tajes, M. (1) 273-288

Murayama, S., see Miyashita (4) 1031-1038

Murchison, C., see Harris, C.J. (1) 179-192

Murray, I.V.J., see Coskuner, O. (2) 561-574

Na, D.L., see Kim, G.H. (1) 169-178

Nacmias, B., see Evangelisti, E. (1) 289-300

Naffa, R., see Al-khateeb, E. (1) 203-211

Någren, K., see Scheinin, N.M. (1) 193-202

Naismith, S.L., see Mowszowski, L. (4) 1095-1108

Nakagawa, R., see Miyashita (4) 1031-1038

Nakashima, K., see Miyashita (4) 1031-1038

Nakata, K., see Miyashita (4) 1031-1038

Namer, I.J., see Blanc, F. (4) 1087-1093

Nelson, L., P. Gard and N. Tabet, Hypertension and Inflammation in Alzheimer's Disease: Close Partners in Disease Development and Progression! (2) 331-343

Neville, C., see Cohen-Mansfield, J. (1) 15-28

Ng, K.S., see Ka-Fung Mak, H. (3) 749-758

Ng, T.P., L. Feng, K.B. Yap, T.S. Lee, C.H. Tan and B. Winblad, Long-Term Metformin Usage and Cognitive Function among Older Adults with Diabetes (1) $61-68$

Ni, J., see Song, G. (1) 85-99

Ning, L.-N., see Lin, L. (4) 1149-1163

Nishizawa, M., see Miyashita (4) 1031-1038

Nogueira, M.L., see Dourado, M.C.N. (3) 947-956

Norrie, L., see Mowszowski, L. (4) 1095-1108

Oh, M.S., see Moon, M. (2) 599-613

Ohrui, T., see Waragai, M. (4) 1207-1222

Olde Rikkert, M.G.M., F.R. Verhey, J.W.C. Sijben, F.H. Bouwman, P.L.J. Dautzenberg, M. Lansink, W.M.W. Sipers, D.Z.B. van Asselt, A.M.J. van Hees, M. Stevens, B. Vellas and P. Scheltens, Differences in Nutritional Status Between Very Mild Alzheimer's Disease Patients and Healthy Controls (1) 261-271

Olde Rikkert, M.G.M., see Hartmann, T. (3) 715-717

Olives, J., see Rami, L. (2) 453-466

Olivier, J.-L., see Sauvée, M. (2) 377-386

Ono, K., see Wang, J. (2) 643-650

Ooya, T., see Miyashita (4) 1031-1038

Ossenkoppele, R., see Zwan, M. (3) 801-807

Padilla-Martínez, I.I., see Hernández-Rodríguez, M. (4) 1073-1085

Padovani, A., see Borroni, B. (2) 371-376
Padovani, A., see Testi, S. (3) 709-714

Pai, M.-C., see Shih, Y.-H. (3) 855-865

Palo, G.D., see Gareri, P. (2) 633-640

Pang, X.-B., see Yu, Y.-Z. (1) 243-260

Pappatà, S., see Testi, S. (3) 709-714

Parnetti, L., see Borroni, B. (2) 371-376

Pasinetti, G.M., see Wang, J. (2) 643-650

Patel, A.B., see Tiwari, V. (2) 387-399

Patrone, C., see Hussain, S. (2) 551-560

Paxinos, G., see Fu, Y. (4) 1177-1192

Peluso, S., see Testi, S. (3) 709-714

Peña-Casanova, J., see Sánchez-Benavides, G. (3) 887-901

Pepe, D., see Borroni, B. (2) 371-376

Perola, M., see Scheinin, N.M. (1) 193-202

Perret-Liaudet, A., see Xie, J. (4) 1109-1116

Perri, R., see Borroni, B. (2) 371-376

Pfeifer, A., see Froestl, W. (4) 961-1019

Philippi, N., see Blanc, F. (4) 1087-1093

Phillips, J.E., see Tales, A. (3) 655-661

Phillips, J.E., see Tales, A. (3) 665-666

Piermartiri, T.C., see de Oliveira, J. (1) 43-60

Piguet, O., see Leyton, C.E. (2) 575-585

Pijnenburg, Y.A.L., see van der Flier, W.M. (1) 313-327

Pinton, S., see de Oliveira, J. (1) 43-60

Pisa, D., see Alonso, R. (1) 301-311

Praticò, D., see Chu, J. (3) 729-737

Prediger, R.D.S., see de Oliveira, J. (1) 43-60

Prins, N., see van der Flier, W.M. (1) 313-327

Provinciali, L., see Viticchi, G. (2) 401-410

Putignano, D., see Gareri, P. (2) 633-640

Putignano, S., see Gareri, P. (2) 633-640

Qi, J.-S., see Qiao, F. (4) 1165-1176

Qian, W., see Ka-Fung Mak, H. (3) 749-758

Qiao, F., X.-P. Gao, L. Yuan, H.-Y. Cai and J.-S. Qi, Apolipoprotein E4 Impairs in vivo Hippocampal Long-Term Synaptic Plasticity by Reducing the Phosphorylation of CaMKII $\alpha$ and CREB (4) 1165-1176

Qin, C., see Zhang, L. (4) 1193-1205

Qiu, W.-Y., see Yu, Y.-Z. (1) 243-260

Qu, J., see Yang, M. (4) 1117-1129

Quaranta, D., see Vecchio, F. (1) 113-127

Quinn, J.F., see Harris, C.J. (1) 179-192

Rábano, A., see Alonso, R. (1) 301-311

Rainero, I., see Borroni, B. (2) 371-376

Ralle, M., see Harris, C.J. (1) 179-192

Rami, L., M.A. Mollica, C. García-Sanchez, J. Saldaña, B. Sanchez, I. Sala, C. Valls-Pedret, M. Castellví, 
J. Olives and J.L. Molinuevo, The Subjective Cognitive Decline Questionnaire (SCD-Q): A Validation Study (2) 453-466

Ramos-Fernández, E., see Tajes, M. (1) 273-288

Recillas-Targa, F., see Cadena-del-Castillo, C. (3) 845854

Rektorova, I., see Krajcovicova, L. (4) 1229-1238

Religa, D., see Eriksson, H. (2) 411-419

Religa, D., see Garcia-Ptacek, S. (2) 467-477

Rentz, D.M., see Marshall, G.A. (3) 719-728

Rhoads, A., see Harris, C.J. (1) 179-192

Rinne, J.O., see Scheinin, N.M. (1) 193-202

Rizak, J., see Yang, M. (4) 1117-1129

Rizak, J., see Yang, M. (4) 1131-1147

Robinson, E., see Harris, C.J. (1) 179-192

Robinson, L., see Siervo, M. (1) 151-161

Robles, A., see Sánchez-Benavides, G. (3) 887-901

Roca, I., see Alegret, M. (3) 739-748

Rodriguez, C., see Haller, S. (1) 101-112

Rogers, E., see Shea, T.B. (3) 667-669

Rokka, J., see Scheinin, N.M. (1) 193-202

Romero, J.P., J. Benito-León, E.D. Louis and F. Bermejo-Pareja, Under Reporting of Dementia Deaths on Death Certificates: A Systematic Review of Population-based Cohort Studies (1) 213-221

Rosales-Hernández, M.C., see Hernández-Rodríguez, M. (4) 1073-1085

Rose, K., see Cohen-Mansfield, J. (1) 15-28

Rosen, A.C., Is There More to Subjective Cognitive Impairment than Meets the Eye? Obligations and Opportunities (3) 663-664

Rosende-Roca, M., see Alegret, M. (3) 739-748

Rossini, P.M., see Vecchio, F. (1) 113-127

Rossini, P.M., see Vecchio, F. (4) 1239-1249

Roullet-Solignac, I., see Xie, J. (4) 1109-1116

Rubio-Moscardó, F., see Tajes, M. (1) 273-288

Rudrabhatla, P., Regulation of Neuronal Cytoskeletal Protein Phosphorylation in Neurodegenerative Diseases (3) 671-684

Ruíz, A., see Alegret, M. (3) 739-748

Russell, C.L., S. Koncarevic and M.A. Ward, PostTranslational Modifications in Alzheimer's Disease and the Potential for New Biomarkers (2) 345-364

Russo, C.V., see Testi, S. (3) 709-714

Rusznák, Z., see Fu, Y. (4) 1177-1192

Sáez-Valero, J., see Campanari, M.-L. (3) 911-924

Saito, Y., see Miyashita (4) 1031-1038

Sala, I., see Rami, L. (2) 453-466
Saldaña, J., see Rami, L. (2) 453-466

Sanchez, B., see Rami, L. (2) 453-466

Sánchez-Benavides, G., J. Peña-Casanova, M. CasalsColl, N. Gramunt, J.L. Molinuevo, B. GómezAnsón, M. Aguilar, A. Robles, C. Antúnez, C. Martínez-Parra, A. Frank-García, M. FernándezMartínez and R. Blesa for the NEURONORMA Study Team, Cognitive and Neuroimaging Profiles in Mild Cognitive Impairment and Alzheimer's Disease: Data from the Spanish Multicenter Normative Studies (NEURONORMA Project) (3) 887-901

Santos, R.L., see Dourado, M.C.N. (3) 947-956

Sasaki, K., see Miyashita (4) 1031-1038

Sauvée, M., G. DidierLaurent, C. Latarche, M.-C. Escanyé, J.-L. Olivier and C. MalaplateArmand, Additional Use of $A \beta_{42} / A \beta_{40}$ Ratio with Cerebrospinal Fluid Biomarkers P-Tau and $A \beta_{42}$ Increases the Level of Evidence of Alzheimer's Disease Pathophysiological Process in Routine Practice (2) 377-386

Savage, S., see Leyton, C.E. (2) 575-585

Scheinin, N.M., K. Wikman, A. Jula, M. Perola, T. Vahlberg, J. Rokka, K. Någren, M. Viitanen and J.O. Rinne, Cortical ${ }^{11} \mathrm{C}$-PIB Uptake is Associated with Age, APOE Genotype, and Gender in "Healthy Aging" (1) 193-202

Scheltens, P., see Hartmann, T. (3) 715-717

Scheltens, P., see Olde Rikkert, M.G.M. (1) 261-271

Scheltens, P., see van der Flier, W.M. (1) 313-327

Scheltens, P., see Zwan, M. (3) 801-807

Schubert, S., see Leyton, C.E. (2) 575-585

Sellal, F., see Blanc, F. (4) 1087-1093

Serikawa, K., see Miyashita (4) 1031-1038

Shafagoj, Y., see Al-khateeb, E. (1) 203-211

Shao, H., Y. Zhang, Y. Dong, B. Yu, W. Xia and Z. Xie, Chronic Treatment with Anesthetic Propofol Improves Cognitive Function and Attenuates Caspase Activation in Both Aged and Alzheimer's Disease Transgenic Mice (2) 499-513

Shapira, J., see Lee, G.J. (3) 793-800

Shaw, L.M., see Korecka, M. (2) 441-451

Shea, T.B. and E. Rogers, Has Prenatal Folate Supplementation Established an At-Risk Population for Age-Related Cognitive Decline? (3) 667-669

Shea, T.B., see Taddeo, M.A. (2) 365-369

Shen, Y., see Sun, Q. (3) 877-886

Sheng, S.-1., see Zhang, L. (4) 1193-1205

Shi, Q., see Song, G. (1) 85-99 
Shiesh, S.-C., see Shih, Y.-H. (3) 855-865

Shih, Y.-H., K.-J. Tsai, C.-W. Lee, S.-C. Shiesh, W.-T.

Chen, M.-C. Pai and Y.-M. Kuo, Apolipoprotein

$\mathrm{C}$-III is an Amyloid- $\beta$-Binding Protein and an Early Marker for Alzheimer's Disease (3) 855-865

Shoji, M., see Miyashita (4) 1031-1038

Siervo, M., S.L. Harrison, C. Jagger, L. Robinson and B.C.M. Stephan, Metabolic Syndrome and Longitudinal Changes in Cognitive Function: A Systematic Review and Meta-Analysis (1) 151-161

Sijben, J.W.C., see Hartmann, T. (3) 715-717

Sijben, J.W.C., see Olde Rikkert, M.G.M. (1) 261-271

Silvestrini, M., see Viticchi, G. (2) 401-410

Simmons, A., see Falahati, F. (3) 685-708

Simó, C., see Lodeiro, M. (1) 223-232

Simone, M., see Gareri, P. (2) 633-640

Sinanaj, I., see Haller, S. (1) 101-112

Sipers, W.M.W., see Olde Rikkert, M.G.M. (1) 261-271

Sjöholm, Å., see Hussain, S. (2) 551-560

Skaane, N.V., see Engvig, A. (3) 779-791

Soininen, H., see Hartmann, T. (3) 715-717

Song, F., see Feng, X. (2) 587-597

Song, F., see Sun, J. (4) 1039-1056

Song, G., Z. Zhang, L. Wen, C. Chen, Q. Shi, Y. Zhang, J. Ni and Q. Liu, Selenomethionine Ameliorates Cognitive Decline, Reduces Tau Hyperphosphorylation, and Reverses Synaptic Deficit in the Triple Transgenic Mouse Model of Alzheimer's Disease (1) 85-99

Song, Y.-Q., see Ka-Fung Mak, H. (3) 749-758

Sotolongo, O., see Alegret, M. (3) 739-748

Sousa, M.F.B., see Dourado, M.C.N. (3) 947-956

Stam, C.J., see van der Flier, W.M. (1) 313-327

Stang, A., see Dlugaj, M. (2) 479-497

Stephan, B.C.M., see Siervo, M. (1) 151-161

Stevens, M., see Olde Rikkert, M.G.M. (1) 261-271

Struyfs, H., J.L. Molinuevo, J.-J. Martin, P.P. De Deyn and S. Engelborghs, Validation of the AD-CSFIndex in Autopsy-Confirmed Alzheimer's Disease Patients and Healthy Controls (3) 903-909

Suga, M., see Miyashita (4) 1031-1038

Sui, X.-1., see Zhang, L. (4) 1193-1205

Sun, J., F. Song, J. Wang, G. Han, Z. Bai, B. Xie, X. Feng, J. Jia, Y. Duan and H. Lei, Hidden Risk Genes with High-Order Intragenic Epistasis in Alzheimer's Disease (4) 1039-1056

Sun, J., see Feng, X. (2) 587-597

Sun, Q., H. Hampel, K. Blennow, S. Lista, A. Levey, B. Tang, R. Li and Y. Shen, Increased Plasma TACE Activity in Subjects with Mild Cognitive
Impairment and Patients with Alzheimer's Disease (3) $877-886$

Sun, Z.-W., see Yu, Y.-Z. (1) 243-260

Sundseth, Ø., see Engvig, A. (3) 779-791

Suzuki, T., see Tajes, M. (1) 273-288

Suzuki, T., see Waragai, M. (4) 1207-1222

Szigeti, K., B. Kellermayer, J.M. Lentini, B. Trummer, D. Lal, R.S. Doody, L. Yan, S. Liu, C. Ma and The Texas Alzheimer Research and Care Consortium, Ordered Subset Analysis of Copy Number Variation Association with Age at Onset of Alzheimer's Disease (4) 1063-1071

Tabet, N., see Nelson, L. (2) 331-343

Taddeo, M.A., S. Lee and T.B. Shea, Synergistic Inhibition of Synaptic Signaling in Cortical Cultures by Subcytotoxic Levels of Oligomerized Amyloid- $\beta$ and Iron: Alleviation by Zinc (2) 365 369

Taipale, H., M. Koponen, A. Tanskanen, A.-M. Tolppanen, J. Tiihonen and S. Hartikainen, Antipsychotic Polypharmacy among a Nationwide Sample of Community-Dwelling Persons with Alzheimer's Disease (4) 1223-1228

Tajes, M., A. Eraso-Pichot, F. Rubio-Moscardó, B. Guivernau, E. Ramos-Fernández, M. BoschMorató, F.X. Guix, J. Clarimón, G.P. Miscione, M. Boada, G. Gil-Gómez, T. Suzuki, H. Molina, J. Villà-Freixa, R. Vicente and F.J. Muñoz, Methylglyoxal Produced by Amyloid- $\beta$ Peptide-Induced Nitrotyrosination of Triosephosphate Isomerase Triggers Neuronal Death in Alzheimer's Disease (1) 273-288

Takahashi, H., see Miyashita (4) 1031-1038

Takahashi, M., see Miyashita (4) 1031-1038

Takao, T., see Miyashita (4) 1031-1038

Takeda, M., see Miyashita (4) 1031-1038

Tales, A., G.K. Wilcock, J.E. Phillips and A. Bayer, Is There More to Subjective Cognitive Impairment than Meets the Eye? A Perspective (3) 655-661

Tales, A., G.K. Wilcock, J.E. Phillips and A. Bayer, Is There More to Subjective Cognitive Impairment than Meets the Eye? Raising Awareness (3) 665666

Tan, C.-C., J.-T. Yu, H.-F. Wang, M.-S. Tan, X.-F. Meng, C. Wang, T. Jiang, X.-C. Zhu and L. Tan, Efficacy and Safety of Donepezil, Galantamine, Rivastigmine, and Memantine for the Treatment of Alzheimer's Disease: A Systematic Review and Meta-Analysis (2) 615-631 
Tan, C.H., see Ng, T.P. (1) 61-68

Tan, L., see Tan, C.-C. (2) 615-631

Tan, M.-S., see Tan, C.-C. (2) 615-631

Tang, B., see Sun, Q. (3) 877-886

Tanskanen, A., see Taipale, H. (4) 1223-1228

Tao, J.-j., see Zhang, L. (4) 1193-1205

Tárraga, L., see Alegret, M. (3) 739-748

Tarumi, T., D.I. Dunsky, M.A. Khan, J. Liu, C. Hill, K. Armstrong, K. Martin-Cook, C.M. Cullum and R. Zhang, Dynamic Cerebral Autoregulation and Tissue Oxygenation in Amnestic Mild Cognitive Impairment (3) 765-778

Tasca, C.I., see de Oliveira, J. (1) 43-60

Teipel, S.J., M.J. Grothe, M. Filippi, A. Fellgiebel, M. Dyrba, G.B. Frisoni, T. Meindl, A.L.W. Bokde, H. Hampel, S. Klöppel, K. Hauenstein and the EDSD study group, Fractional Anisotropy Changes in Alzheimer's Disease Depend on the Underlying Fiber Tract Architecture: A Multiparametric DTI Study using Joint Independent Component Analysis (1) 69-83

Teschler, H., see Dlugaj, M. (2) 479-497

Testi, S., S. Peluso, G.M. Fabrizi, A. Antenora, C.V. Russo, S. Pappatà, A. Padovani, M. Ferrarini and A. Filla, A Novel PSEN1 Mutation in a Patient with Sporadic Early-Onset Alzheimer's Disease and Prominent Cerebellar Ataxia (3) 709-714

Teunissen, C., see Zwan, M. (3) 801-807

Teunissen, C.E., see van der Flier, W.M. (1) 313-327

Thaler, C., see Brandner, S. (4) 1057-1062

Thompson, P.M., see Lee, G.J. (3) 793-800

Tian, Q., see Lin, L. (4) 1149-1163

Tiihonen, J., see Taipale, H. (4) 1223-1228

Tiwari, V. and A.B. Patel, Pyruvate Carboxylase and Pentose Phosphate Fluxes are Reduced in A $\beta \mathrm{PP}-$ PS1 Mouse Model of Alzheimer's Disease: A ${ }^{13} \mathrm{C}$ NMR Study (2) 387-399

Tokuda, T., see Waragai, M. (4) 1207-1222

Toledo, J.B., see Korecka, M. (2) 441-451

Tolppanen, A.-M., see Taipale, H. (4) 1223-1228

Toma, S., see Haller, S. (1) 101-112

Tomita, N., see Miyashita (4) 1031-1038

Toneff, T., see Hook, G. (1) 129-149

Torkamani, M., L. McDonald, I.S. Aguayo, C. Kanios, M.-N. Katsanou, L. Madeley, P.D. Limousin, A.J. Lees, M. Haritou, M. Jahanshahi and the ALADDIN Collaborative Group, A Randomized Controlled Pilot Study to Evaluate a Technology Platform for the Assisted Living of People with Dementia and their Carers (2) 515-523

Tremolizzo, L., see Borroni, B. (2) 371-376

Trojanowski, J.Q., see Korecka, M. (2) 441-451
Trummer, B., see Szigeti, K. (4) 1063-1071

Tsai, K.-J., see Shih, Y.-H. (3) 855-865

Tsolaki, M., see Mukaetova-Ladinska, E.B. (4) 12511260

Turla, M., see Borroni, B. (2) 371-376

Tzavaras, N., see Wang, J. (2) 643-650

Uemura, K., see Waragai, M. (4) 1207-1222

Urakami, K., see Miyashita (4) 1031-1038

Vahlberg, T., see Scheinin, N.M. (1) 193-202

Valdes-Quezada, C., see Cadena-del-Castillo, C. (3) 845-854

Valero, S., see Alegret, M. (3) 739-748

Valls-Pedret, C., see Rami, L. (2) 453-466

van Asselt, D.Z.B., see Olde Rikkert, M.G.M. (1) 261271

van Berckel, B., see Zwan, M. (3) 801-807

van Berckel, B.N.M., see van der Flier, W.M. (1) 313327

van der Flier, W., see Zwan, M. (3) 801-807

van der Flier, W.M., Y.A.L. Pijnenburg, N. Prins, A.W. Lemstra, F.H. Bouwman, C.E. Teunissen, B.N.M. van Berckel, C.J. Stam, F. Barkhof, P.J. Visser, E. van Egmond and P. Scheltens, Optimizing Patient Care and Research: The Amsterdam Dementia Cohort (1) 313-327

van Egmond, E., see van der Flier, W.M. (1) 313-327

van Harten, A., see Zwan, M. (3) 801-807

van Hees, A.M.J., see Olde Rikkert, M.G.M. (1) 261271

van Wijk, N., see Hartmann, T. (3) 715-717

Varghese, M., see Wang, J. (2) 643-650

Vaskova, O., see Mukaetova-Ladinska, E.B. (4) 12511260

Vecchio, F., F. Miraglia, C. Marra, D. Quaranta, M.G. Vita, P. Bramanti and P.M. Rossini, Human Brain Networks in Cognitive Decline: A Graph Theoretical Analysis of Cortical Connectivity from EEG Data (1) 113-127

Vecchio, F., F. Miraglia, P. Bramanti and P.M. Rossini, Human Brain Networks in Physiological Aging: A Graph Theoretical Analysis of Cortical Connectivity from EEG Data (4) 1239-1249

Vellas, B., see Hartmann, T. (3) 715-717

Vellas, B., see Olde Rikkert, M.G.M. (1) 261-271

Verhey, F.R., see Olde Rikkert, M.G.M. (1) 261-271

Vermeiren, Y., D.V. Dam, T. Aerts, S. Engelborghs and P.P. De Deyn, Brain Region-Specific Monoaminergic Correlates of Neuropsychiatric Symptoms in Alzheimer's Disease (3) 819-833

Vernieri, F., see Viticchi, G. (2) 401-410 
Vicente, R., see Tajes, M. (1) 273-288

Vighetto, A., see Xie, J. (4) 1109-1116

Viitanen, M., see Scheinin, N.M. (1) 193-202

Villà-Freixa, J., see Tajes, M. (1) 273-288

Visser, P.J., see van der Flier, W.M. (1) 313-327

Vita, M.G., see Vecchio, F. (1) 113-127

Viticchi, G., L. Falsetti, F. Vernieri, C. Altamura, R. Altavilla, S. Luzzi, M. Bartolini, L. Provinciali and M. Silvestrini, Apolipoprotein E Genotype and Cerebrovascular Alterations Can Influence Conversion to Dementia in Patients with Mild Cognitive Impairment (2) 401-410

Voss, K., see Harris, C.J. (1) 179-192

Walhovd, K.B., see Engvig, A. (3) 779-791

Waligorska, T., see Korecka, M. (2) 441-451

Wang, C., see Tan, C.-C. (2) 615-631

Wang, H.-F., see Tan, C.-C. (2) 615-631

Wang, J., M. Varghese, K. Ono, M. Yamada, S. Levine, N. Tzavaras, B. Gong, W.J. Hurst, R.D. Blitzer and G.M. Pasinetti, Cocoa Extracts Reduce Oligomerization of Amyloid- $\beta$ : Implications for Cognitive Improvement in Alzheimer's Disease (2) 643-650

Wang, J., see Feng, X. (2) 587-597

Wang, J., see Sun, J. (4) 1039-1056

Wang, J., see Yang, M. (4) 1117-1129

Wang, J., see Yang, M. (4) 1131-1147

Wang, J., see Zhou, X. (3) 925-935

Wang, J.-Z., see Lin, L. (4) 1149-1163

Wang, L., see Lin, L. (4) 1149-1163

Wang, S., see Yu, Y.-Z. (1) 243-260

Wang, T., see Kapila, A.K. (1) 1-13

Wang, W.-B., see Yu, Y.-Z. (1) 243-260

Wang, Y., see Zhou, X. (3) 925-935

Wang, Z., see Yang, M. (4) 1131-1147

Waragai, M., S. Hata, T. Suzuki, R. Ishii, C. Fujii, T. Tokuda, H. Arai, T. Ohrui, S. Higuchi, M. Yoshida, K. Igarashi, M. Moriya, N. Iwai and K. Uemura, Utility of SPM8 plus DARTEL (VSRAD) Combined with Magnetic Resonance Spectroscopy as Adjunct Techniques for Screening and Predicting Dementia due to Alzheimer's Disease in Clinical Practice (4) 1207-1222

Ward, M.A., see Russell, C.L. (2) 345-364

Ward, P.B., see Mowszowski, L. (4) 1095-1108

Watanabe, K., see Miyashita (4) 1031-1038

Watts, H.R., see Kapila, A.K. (1) 1-13

Wege, N., see Dlugaj, M. (2) 479-497

Weimar, C., see Dlugaj, M. (2) 479-497

Weinreich, G., see Dlugaj, M. (2) 479-497
Welge, J., see Boespflug, E.L. (2) 421-430

Wen, L., see Song, G. (1) 85-99

Wen, Y., see Miyashita (4) 1031-1038

Wessendorf, T.E., see Dlugaj, M. (2) 479-497

Westlye, L.T., see Engvig, A. (3) 779-791

Westman, E., see Falahati, F. (3) 685-708

Wierenga, C.E., see Zlatar, Z.Z. (3) 809-817

Wikman, K., see Scheinin, N.M. (1) 193-202

Wilcock, G.K., see Tales, A. (3) 655-661

Wilcock, G.K., see Tales, A. (3) 665-666

Winblad, B., see Ng, T.P. (1) 61-68

Winkler, A., see Dlugaj, M. (2) 479-497

Wu, J., see Zhang, L. (4) 1193-1205

Wu, S., see Yang, M. (4) 1131-1147

Wurtman, R.J., see Hartmann, T. (3) 715-717

Xia, W., see Shao, H. (2) 499-513

Xie, B., see Feng, X. (2) 587-597

Xie, B., see Sun, J. (4) 1039-1056

Xie, J., A. Gabelle, A. Dorey, A. Garnier-Crussard, A. Perret-Liaudet, F. Delphin-Combe, A. Bathsavanis, V. Dauphinot, S. Lehmann, B. Mercier, V. Desestret, I. Roullet-Solignac, A. Vighetto and P. Krolak-Salmon, Initial Memory Deficit Profiles in Patients with a Cerebrospinal Fluid Alzheimer's Disease Signature (4) 1109-1116

Xie, Z., see Shao, H. (2) 499-513

Xu, Q., see Yu, Y.-Z. (1) 243-260

Xu, Y.-f., see Zhang, L. (4) 1193-1205

Yamada, M., see Miyashita (4) 1031-1038

Yamada, M., see Wang, J. (2) 643-650

Yamaguchi, H., see Miyashita (4) 1031-1038

Yan, L., see Szigeti, K. (4) 1063-1071

Yang, J., see Yang, M. (4) 1117-1129

Yang, J., see Yang, M. (4) 1131-1147

Yang, M., J. Lu, J. Miao, J. Rizak, J. Yang, R. Zhai, J. Zhou, J. Qu, J. Wang, S. Yang, Y. Ma, X. Hu and R. He, Alzheimer's Disease and Methanol Toxicity (Part 1): Chronic Methanol Feeding Led to Memory Impairments and Tau Hyperphosphorylation in Mice (4) 1117-1129

Yang, M., J. Miao, J. Rizak, R. Zhai, Z. Wang, T. Huma, T. Li, N. Zheng, S. Wu, Y. Zheng, X. Fan, J. Yang, J. Wang, S. Yang, Y. Ma, L. Lü, R. He and X. Hu, Alzheimer's Disease and Methanol Toxicity (Part 2): Lessons from Four Rhesus Macaques (Macaca mulatta) Chronically Fed Methanol (4) 1131-1147

Yang, S., see Yang, M. (4) 1117-1129

Yang, S., see Yang, M. (4) 1131-1147

Yang, S.-S., see Lin, L. (4) 1149-1163 
Yao, Z.-g., see Zhang, L. (4) 1193-1205

Yap, K.B., see Ng, T.P. (1) 61-68

Yoshida, M., see Waragai, M. (4) 1207-1222

Yoshimoto, S., see Miyashita (4) 1031-1038

Yu, B., see Shao, H. (2) 499-513

Yu, J., see Hook, G. (1) 129-149

Yu, J.-T., see Tan, C.-C. (2) 615-631

Yu, Y.-Z., W.-B. Wang, A. Chen, Q. Chang, S. Liu, M. Zhao, S. Wang, W.-Y. Qiu, X.-B. Pang, Q. Xu and Z.-W. Sun, Strikingly Reduced Amyloid Burden and Improved Behavioral Performance in Alzheimer's Disease Mice Immunized with Recombinant Chimeric Vaccines by Hexavalent Foldable $\mathrm{A} \beta_{1-15}$ Fused to Toxin-Derived Carrier Proteins (1) 243-260

Yuan, L., see Qiao, F. (4) 1165-1176

Yuzuriha, T., see Miyashita (4) 1031-1038

Zambenedetti, P., see Bolognin, S. (1) 29-42

Zampagni, M., see Evangelisti, E. (1) 289-300

Zanetti, O., see Borroni, B. (2) 371-376

Zatta, P., see Bolognin, S. (1) 29-42

Zhai, R., see Yang, M. (4) 1117-1129

Zhai, R., see Yang, M. (4) 1131-1147

Zhang, J., see Zhou, X. (3) 925-935

Zhang, L., C. Liu, J. Wu, J.-j. Tao, X.-1. Sui, Z.-g. Yao, Y.-f. Xu, L. Huang, H. Zhu, S.-1. Sheng and C. Qin, Tubastatin A/ACY-1215 Improves Cognition in Alzheimer's Disease Transgenic Mice (4) 11931205

Zhang, R., see Tarumi, T. (3) 765-778

Zhang, T., see Lin, L. (4) 1149-1163
Zhang, Y., see Shao, H. (2) 499-513

Zhang, Y., see Song, G. (1) 85-99

Zhang, Z., see Song, G. (1) 85-99

Zhang, Z., see Zhou, X. (3) 925-935

Zhao, H., see Zhao, Y. (3) 835-844

Zhao, M., see Yu, Y.-Z. (1) 243-260

Zhao, Y., H. Zhao, N. Lobo, X. Guo, S.M. Gentleman and D. Ma, Celastrol Enhances Cell Viability and Inhibits Amyloid- $\beta$ Production Induced by Lipopolysaccharide In Vitro (3) 835-844

Zheng, N., see Yang, M. (4) 1131-1147

Zheng, Y., see Yang, M. (4) 1131-1147

Zhou, J., see Yang, M. (4) 1117-1129

Zhou, X., J. Zhang, Y. Chen, T. Ma, Y. Wang, J. Wang and Z. Zhang, Aggravated Cognitive and Brain Functional Impairment in Mild Cognitive Impairment Patients with Type 2 Diabetes: A Resting-State Functional MRI Study (3) 925-935

Zhu, H., see Zhang, L. (4) 1193-1205

Zhu, X.-C., see Tan, C.-C. (2) 615-631

Zlatar, Z.Z., C.E. Wierenga, K.J. Bangen, T.T. Liu and A.J. Jak, Increased Hippocampal Blood Flow in Sedentary Older Adults at Genetic Risk for Alzheimer's Disease (3) 809-817

Zurlo, A., see Gareri, P. (2) 633-640

Zwan, M., A. van Harten, R. Ossenkoppele, F. Bouwman, C. Teunissen, S. Adriaanse, A. Lammertsma, P. Scheltens, B. van Berckel and W. van der Flier, Concordance Between Cerebrospinal Fluid Biomarkers and $\left[{ }^{11} \mathrm{C}\right]$ PIB PET in a Memory Clinic Cohort (3) 801-807 